\title{
Demencia y cognición social: su relación con las redes de apoyo social ${ }^{10}$
}

\author{
John Alexander Castro Muñoz \\ Magister en Psicología \\ Universidad Piloto de Colombia \\ Bogotá \\ Correo electrónico: alexandercastro.psico@gmail.com \\ Yesika Paola Rincón Currea \\ Psicóloga \\ Universidad Piloto de Colombia \\ Bogotá \\ Correo electrónico: yesikarinconc@gmail.com
Yeraldin Fernanda Farfán Díaz
Psicóloga
Universidad Piloto de Colombia
Bogotá \\ Correo electrónico: yeraldin_farfan_diaz@hotmail.com
}

\author{
Andrea Ramírez Poveda \\ Psicóloga \\ Universidad Piloto de Colombia \\ Bogotá \\ Correo electrónico: andre-829@hotmail.com
}

Recibido: $23 / 05 / 2018$

Evaluado: 05/09/2018

Aceptado: 03/12/2018

\section{Resumen}

La demencia tipo Alzheimer (DTA) y la demencia fronto-temporal (DFT), desde una perspectiva psicosocial, coinciden, debido a sus características, en el impacto directo y significativo sobre los integrantes del entorno social de quien las vivencia. Objetivo: Identificar y describir a partir de la información disponible en la literatura, el papel de las redes de apoyo social, enfatizando en el cuidador, considerando en éste, los procesos de la cognición social, unicamente a partir del análisis conceptual y de evidencias empíricas disponibles, procedentes de investigaciones en torno a estos dos tipos de demencias. Método: Revisión documental y sistemática de investigaciones procedentes de bases de datos especializadas. Resultados: se evidenciaron diferencias en el nivel de impacto que ambos tipos de demencias tienen sobre la red de apoyo social, incluido el cuidador ${ }^{1}$, dependiendo del tipo de demencia, y en función de la disponibilidad de recursos por parte de la red. Así mismo, se observó que existe un vacío documental en el análisis acerca de la participación de la cognición social en la relación entre los integrantes de la red y aquellas personas con alguna de estas demencias. De esta investigación surgen interrogantes encaminados que sugieren profundizar más allá en la descripción de estos tipos de demencia.

Palabras clave

Alzheimer, Demencia, Cognición social, Apoyo social.

10 Para citar este artículo: Castro, J., Rincón, Y., Farfán, Y. \& Ramírez, A. (2019). Demencia y cognición social: su relación con las redes de apoyo social. Informes Psicológicos, 19(2), pp. 143-161http://dx.doi.org/10.18566/infpsic. v19n2a010

1 Persona que atiende de forma permanente las necesidades físicas y emocionales de un enfermo, papel por lo general cumplido por integrantes del núcleo familiar o cercanos y significativos al mismo (Brenda \& ALvarado, 2009). 


\section{Dementia and social cognition: their relationship with social support networks}

Abstract

Due to their characteristics, Alzheimer's dementia (AD) and fronto-temporal dementia (FTD) coincide, from a psychosocial perspective, in the direct and significant impact on the members of the social environment of those who experience them. Objective: to identify and describe the role of social support networks from the information available in the literature, emphasizing the caregiver and considering in them the processes of social cognition, only from conceptual analysis and empirical evidence available from previous research on these two types of dementia. Method: documentary and systematic review of investigations from specialized databases. Results: there were differences in the level of impact that both types of dementia have on the social support network, including the caregiver, depending on the type of dementia and on the availability of resources by the network. Likewise, it was observed that there is a documentary gap in the analysis about the participation of social cognition in the relationship between the members of the network and those suffering from any of these dementias. From this study, arise questions that suggest further description of these types of dementia should be made.

Keywords Alzheimer's, dementia, social cognition, social support.

\section{Demência e cognição social: Sua relação com as redes de suporte social}

Resumo

A demência tipo Alzheimer (DTA) e a demência fronto-temporal (DFT), partindo desde uma perspetiva psico-social, coincidem, devido a suas caraterísticas, no impacto direto e significativo nos integrantes do entorno social daquele que as experimenta. Objetivo: identificar e descrever partindo da informação disponível na literatura, o papel das redes de suporte enfatizando no cuidador, considerando neste os processos de cognição social, exclusivamente partindo da análise conceitual e das evidências empíricas disponíveis decorrentes de pesquisas que tratam destes dois tipos de demências. Método: Revisão documental e sistemática das investigações provenientes de bases de dados especializadas. Resultados: evidenciaram-se diferencias no nível de impacto que ambos tipos de demências têm sobre a rede de suporte social, incluído o cuidador, dependendo do tipo de demência, e em função da disponibilidade de recursos da parte da rede. Também, observouse que há um vácuo documental nas análises ao respeito da participação da cognição social na relação entre os integrantes da rede e aquelas pessoas que possuem alguma destas demências. Desta investigação surgem interrogantes encaminhadas que sugestionam um aprofundamento maior na descrição destes tipos de demência.

Palavras chave Alzheimer, demência, cognição social, suporte social. 


\section{ntroducción}

La Demencia Tipo Alzheimer (DTA), así como la demencia fronto-temporal (DFT), han sido reconocidas como los dos tipos de demencia cortical responsables de la mayoría de casos con impacto degenerativo. La DTA es responsable de aproximadamente el $50 \%$ del total de casos de demencias, mientras la DFT ocupa un 10\% en esta población. La edad de la mayoría de sujetos con DTA se encuentra entre los 60 y 69 años, y con DFT entre los 70 y 79 años (Charro, Diéguez, \& González, 2010; Ostrosky \& Lozano, 2012; Ruiz, Nariño \& Muñoz, 2010)

De acuerdo con la Federación Internacional de Alzheimer (ADI, 2016), en 2015 se estimó que 47 millones de personas presentaban diagnóstico de demencia a nivel mundial y se espera que para 2050 la prevalencia de esta enfermedad haya incrementado a 131 millones de personas. El mayor costo de esta enfermedad es el que se da a nivel humano. De acuerdo con Iragorri (2007), Llibre y Gutiérrez (2014), Méndez (2010) y Ostrosky y Lozano (2012), las familias y cuidadores son quienes sufren las consecuencias psicológicas, físicas, sociales y financieras. Este panorama se presenta especialmente en países en vías de desarrollo. En las cifras presentadas por ADI (2013), en 2013 el 57.7\% de las personas con demencia vivían en países con ingresos bajos o medios, proporción que se espera incremente a 63.4\% en 2030; los índices de prevalencia en América Latina son aproximadamente del $6.5 \%$.
La etapa o el grado de avance en el que se encuentre la enfermedad generan variaciones en la necesidad de acompañamiento requerido por parte del cuidador hacia el paciente. En Colombia, las personas cuidadores presentan necesidades insatisfechas la mayor parte del tiempo, lo que repercute en una sobrecarga, estrés, aislamiento y problemas de salud (Cerquera, Granados \& Buitrago, 2015; Cerquera, Pabón \& Uribe, 2012; Cerquera \& Galvis, 2014; Moreno, Arango \& Rogers, 2010). Es importante para la psicología la profundización en este tema, ya que, dadas las características de las demencias y la carga consecuente en el cuidador, el sistema familiar, a su vez, se ve afectado. La intervención desde la psicología, de acuerdo con ello, tendría que considerar simultáneamente los niveles explicativos de orden: neuropsicológico, clínico y psicosocial. De esta forma podría contar con más y mejores recursos para la intervención sobre los cuidadores, las familias e instituciones involucradas.

El objetivo de la presente investigación consiste en indagar el análisis que se ha hecho sobre el cuidador y los integrantes de la red de apoyo, ante la existencia e interacción con un integrante diagnosticado con alguna de estas demencias, así como el papel que se ha dado a la cognición social en las investigaciones. A través de esta revisión documental se busca dar respuesta a partir de la evidencia disponible, a la pregunta de investigación acerca de: ¿Qué papel ha tenido el cuidador y la cognición social en el abordaje de las Demencias (específicamente DTA y DFT)? 


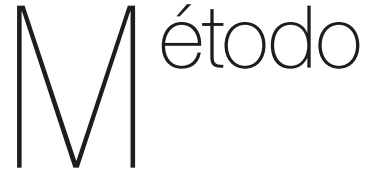

La presente es una investigación exploratoria de tipo documental. Inicialmente se realizó una revisión exhaustiva de la evidencia conceptual y empírica acerca de los dos tipos de demencia (DTA y DFT) en torno a tres ejes explicativos: (a) las funciones cognitivas afectadas: referidas a aquellas actividades mentales que permiten el procesamiento de la información, su adquisición, almacenamiento, recuperación y uso (Rivas, 2008); (b) las estructuras cerebrales implicadas: entendidas como aquellas estructuras en las que está organizado de forma compleja el sistema nervioso central, más específicamente a la organización espacial tridimensional o bloques funcionales que interactúan entre sí para desempeñar funciones particulares (Arango \& Pimienta, 2004); c) Bases genéticas: Información obtenida a partir de la secuencia del ADN en el Genoma Humano relacionada con trastornos a nivel estructural de uno o más genes, que condicionan la aparición de patologías (Defranc, 2011; Ruiz, 2003). Un eje adicional se incluyó, no para la comprensión de las demencias, sino para describir las repercusiones en el entorno. Éste corresponde a un eje (d) que observa el impacto que tendrían estos tipos de demencias, en el funcionamiento de las redes de apoyo de los pacientes, entendiendo a las redes de apoyo de acuerdo con la descripción de Madariaga, Abelló y Sierra (2003), quienes las definen como el lugar en el que se presenta el intercambio dinámico y recíproco entre personas, grupos e instituciones, que posibilita la potenciación -vo maximización - y el enriquecimiento de sus recursos personales, emocionales y sociales. Estos ejes fueron abordados en la selección y revisión de cada una de estas dos demencias. De acuerdo con ello, se aclara que la búsqueda tuvo en cuenta evidencias conceptuales y empíricas publicadas en el periodo comprendido entre el año 2003 y 2016, incluyendo en los buscadores, terminología que permitiera identificar posteriormente al realizar la lectura del contenido de los artículos y documentos, conceptos y evidencias vinculadas a los 4 ejes descritos. Así, se emplearon dentro los buscadores términos como: "Demencia fronto temporal", "Demencia tipo Alzheimer", "Cognición social", "Apoyo social", "Red de apoyo", "Cuidadores", "Empatía" y "Apoyo familiar" entre los principales. Posterior a la identificación de las evidencias a partir de estos buscadores se seleccionaron aquellas que, por su contenido, correspondieran con los 4 ejes. Finalmente, y tras aclarar y reunir suficiente información en cuanto a estos tres ejes, y a partir del análisis de algunos artículos ya revisados (Cerquera et al., 2012; Cerquera \& Galvis, 2014; Moreno et al., 2010), se diseñó la matriz en la cual se organizó la información recolectada de cincuenta (50) artículos publicados entre 2009 y 2017 que han abordado la temática de las demencias y cuidadores, rescatados de bases de datos especializadas como Redalyc, Scielo, Proquest y PsycArticles.

A partir de esta revisión, se espera proyectar un panorama general acerca de factores que se tienen en cuenta durante las investigaciones de demencia DTA y DFT y cuidadores e identificar el papel que se le ha dado a la cognición social en las investigaciones. 


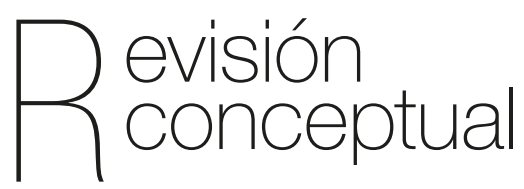

\section{Prevalencia de la Demencia}

De acuerdo con Ostrosky y Lozano (2012), Ruiz et al. (2010) y Gil y Martín (2006), la demencia tipo Alzheimer, así como la demencia fronto-temporal, han sido reconocidas como los tipos primarios de demencia degenerativa primaria -de predominio cortical- en donde la primera es responsable de aproximadamente el $50 \%$ del total de casos de demencias, mientras la DFT ocupa un $10 \%$ entre las demencias; sin embargo, es necesario reconocerla debido a las alteraciones a nivel comportamental que impactan directamente al cuidador (Diehl et al., 2013; Iragorri, 2007; Mioshi et al., 2013). En cuanto a la prevalencia por rango de edad, la DTA es la segunda causa degenerativa de demencias en pacientes de menos de 65 años, mientras la edad media de pacientes con DFT se encuentra entre los 70 a 79 años.

La Federación Internacional de Alzheimer (ADI, 2016) estimó que en 2015 había 46,8 millones de personas con diagnóstico de demencia a nivel mundial y se espera que para 2050 la prevalencia de esta enfermedad haya incrementado a 131,5 millones; estos datos sugieren e invitan a incrementar los esfuerzos para su comprensión, diagnóstico e intervención. Adicionalmente, de acuerdo con ADI (2016), Llibre (2012), Méndez (2010), Ministerio de Salud (MinSalud, 2017) y
Ostrosky y Lozano (2012) este incremento sugiere aumentos en los costos para la salud pública, relacionados con cuidados informales, costos sociales, tratamientos y cuidados médicos; sin embargo, el mayor costo es el que se da a nivel humano, específicamente desde las familias y cuidadores, que son quienes sufren las consecuencias psicológicas, físicas, sociales y financieras. Estas consecuencias incluyen, entre otras, problemas de ansiedad, estrés y la depresión (Iragorri, 2007; Mioshi et al., 2013 y Diehl et al., 2013).

Este panorama se presenta especialmente en países en vías de desarrollo, en los que la prevalencia, como en el caso de América Latina, es aproximadamente del 6.5\%. Así mismo, la tasa media de prevalencia de la demencia en edades mayores o iguales a 60 años en todos los continentes varía de $2.2 \%$ en África hasta $8.9 \%$ en Europa. Respecto a la prevalencia, a comparar entre países, esta cifra se encuentra entre un 1.3\% en la India hasta un $14.9 \%$ en España. La mayoría de las personas afectadas con demencia viven en países en desarrollo. En 2010 el 57.7\% de las personas con demencia vivían en países con ingresos bajos o medios, porcentaje que, de acuerdo a las proyecciones de crecimiento poblacional y envejecimiento, se espera incremente hasta $63.4 \%$ en 2030 (ADI, 2013; Llibre \& Gutiérrez, 2014). Así mismo, MinSalud (2017) reportó que, de acuerdo con un estudio realizado en Colombia, hay una prevalencia de $9.4 \%$ de casos de demencia en mayores de 60 años, sin embargo, en personas mayores de 85 años la prevalencia aumenta hasta el 58\%, siendo mayor su frecuencia en hombres que en mujeres, y mayor en niveles socioeconómicos bajos. 
A continuación, la Figura 1 evidencia la proyección del aumento de la prevalencia de demencia del año 2010 al 2030 de acuerdo a la región; los porcentajes presentados en las cuatro primeras barras de la izquierda hacen referencia a los países desarrollados y el resto a los países en vías de desarrollo.

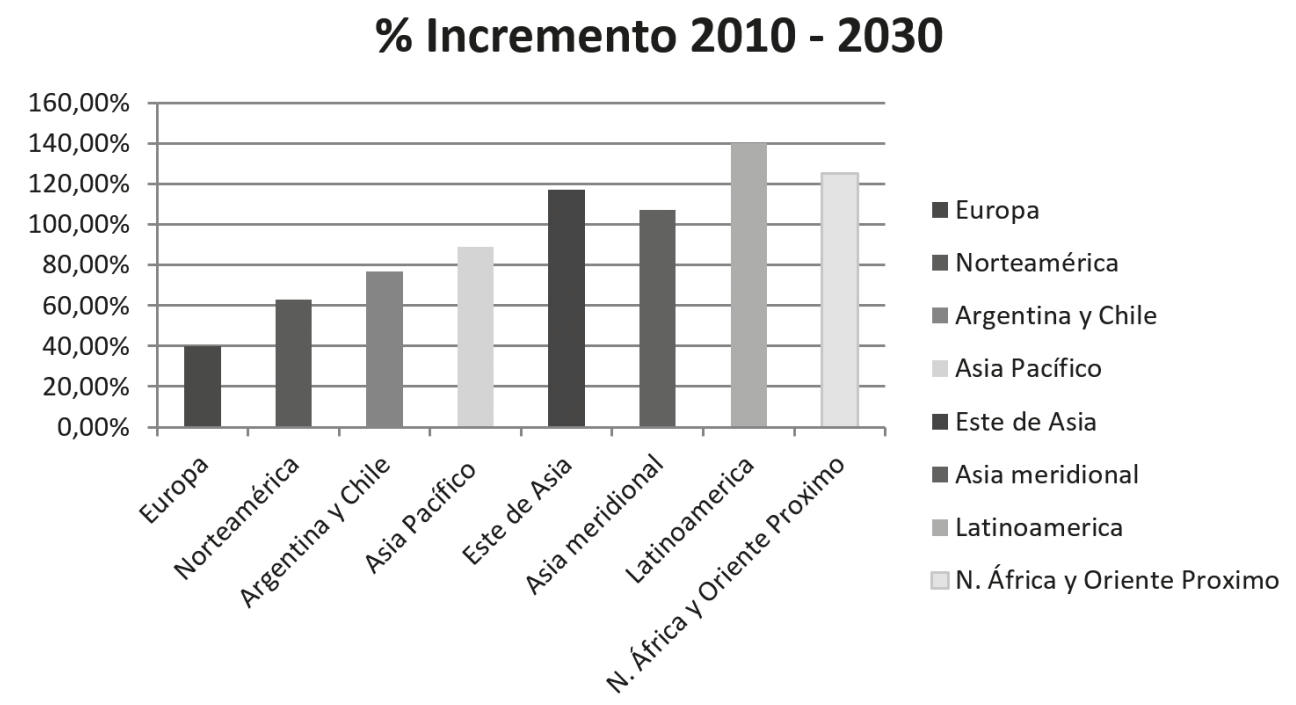

Figura 1. Porcentaje del incremento de la prevalencia de demencia por región.

Fuente: Construida a partir de datos de ADI (2013) x: Región y: Porcentaje de Incremento de prevalencia

Adicionalmente, la etapa o el grado de avance en el que se encuentre la enfermedad genera variaciones en la necesidad de acompañamiento requerido por parte del cuidador hacia el paciente. En Colombia, el número de personas cuidadores presentan necesidades insatisfechas la mayor parte del tiempo, lo que repercute en una sobrecarga, estrés, aislamiento y problemas de salud (Cerquera et al., 2012; Moreno et al., 2010). Además de esto, es importante para la psicología, la profundización en este tema ya que, dadas las características de deterioro cognitivo de las demencias, así como la carga emocional consecuente para el cuidador, la patología afecta el sistema familiar en su totalidad.

\section{Demencia Tipo Alzheimer y Demencia Fronto Temporal}

Como se mencionó anteriormente, para el buen desarrollo de la revisión de investigaciones se profundizó, para explicar y comprender ambas demencias, en tres ejes: estructuras neuropsicológicas, funciones cognitivas y bases genéticas, logrando hacer una comparación entre estos dos tipos de demencia y sus diferentes alteraciones; de esta manera, en la Tabla 1 se presentan las generalidades de lo encontrado en cada uno de los ejes. En este orden, la primera parte hace referencia a la definición de cada 
tipo de demencia, la segunda parte las alteraciones a nivel estructural, la tercera parte a las funciones cognitivas que se ven afectadas durante el desarrollo de la demencia y, finalmente, el material genético que ha sido asociado a la presencia de estos tipos de demencia, a partir de investigaciones.

Tabla 1.

Cuadro comparativo entre Demencia Tipo Alzheimer y Demencia Fronto temporal.

\begin{tabular}{|c|c|c|}
\hline & Demencia Tipo Alzheimer & Demencia Fronto - Temporal \\
\hline 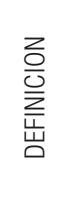 & $\begin{array}{l}\text { "Enfermedad degenerativa de predominio cortical, progre- } \\
\text { siva e irreversible, caracterizada por perdida de diversas } \\
\text { capacidades intelectuales y cognitivas" (Sarasa, 2009, } \\
\text { citado en De la Rubia, Sancho y Cabañés, 2014, p. 132) }\end{array}$ & $\begin{array}{l}\text { "Enfermedad Neurodegenerativa caracterizada por un } \\
\text { cambio progresivo en la personalidad y el comportamiento, } \\
\text { y/o alteración temprana y progresiva del lenguaje, en las } \\
\text { que memoria y capacidades visuoespaciales están relati- } \\
\text { vamente preservadas en estadios iniciales" (Charro et al., } \\
2010, \text { p. 228) }\end{array}$ \\
\hline 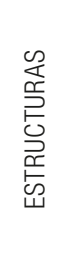 & $\begin{array}{l}\text { Atrofia cerebral global, la mayor afectación tiene lugar en } \\
\text { la corteza cerebral (córtex entorrinal, hipocampo y córtex } \\
\text { parietal), atrofia celular del cerebro basal y mesencéfalo. } \\
\text { Pérdida de neuronas colinérgicas en especial en el núcleo } \\
\text { basal de Meynert, perdida celular en el locus coerelus, de- } \\
\text { crece el sistema noradrenérgico y los núcleos de rafe, que } \\
\text { provoca la disminución de serotonina (Bruna \& Cullell, s.f.) }\end{array}$ & $\begin{array}{l}\text { Zona premotora, área de Broca, CPF derecha, Sistema reti- } \\
\text { cular activador, tálamo, sistema límbico, ganglios basales, } \\
\text { córtex frontal, Tactum, córtex parietal superior, pulvinar } \\
\text { lateral, coliculo superior. Cingulado anterior, CPFDL, neo } \\
\text { estriado, COF, sistema frontal superior, Corteza pre frontal, } \\
\text { porciones dorsolaterales de la CPF, Corteza Fronto Medial, } \\
\text { Corteza Orbito Frontal y Corteza Fronto Medial (Flores \& } \\
\text { Ostrosky-Solís, 2008, p. 49) }\end{array}$ \\
\hline 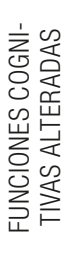 & $\begin{array}{l}\text { Memoria, pensamiento, lenguaje, toma de decisiones, } \\
\text { atención, apraxias, agnosias, afectación en conducta y } \\
\text { personalidad. (Oviedo, Britton \& Villareal, 2016). Atención, } \\
\text { cálculo, funciones viso-espaciales y capacidades ejecuti- } \\
\text { vas, de abstracción y juicio, se preserva la velocidad inte- } \\
\text { lectual y motora gestual (Pérez, 2005; Pérez, 2008) }\end{array}$ & $\begin{array}{l}\text { Se caracteriza por cambios en el comportamiento, en la } \\
\text { personalidad y de las funciones ejecutivas (Charro et al., } \\
\text { 2010), lenguaje (fluidez verbal, disminución en compren- } \\
\text { sión de verbos y sustantivos), atención (sostenida, selec- } \\
\text { tiva, supresión atencional y cambio en foco atencional), } \\
\text { funciones ejecutivas (planificación, flexibilidad, monitoriza- } \\
\text { ción e inhibición de conductas), conducta social (Donoso } \\
\text { \& Salinas, 2009) }\end{array}$ \\
\hline \multirow{5}{*}{ 芯 } & Péptido Beta Amiloide & TAU \\
\hline & Preselina 1 y 2 & Preselina 1 \\
\hline & TAU & GEN PGRNsd \\
\hline & Apolopoproteína & (Lopera, 2012) \\
\hline & $\begin{array}{l}\text { (Granadillo \& Zarante, 2008; } \\
\text { Setó-Salvia \& Clarimón, 2010) }\end{array}$ & \\
\hline
\end{tabular}

Fuente: Elaboración Propia

Tras la profundización en cada eje explicativo, y para que sea posible la comprensión del impacto en las redes de apoyo de pacientes con diagnóstico de estos tipos de demencia, así como el papel que se le ha dado a la cognición social tanto de quienes asumen el rol de cuidadores, como en el desarrollo de las demencias, acorde a lo identificado en las investigaciones disponibles y seleccionadas a partir de los criterios de inclusión previamente descritos, se hace necesario revisar los referentes teóricos que han trabajado en torno a estas dos temáticas. De esta manera, a continuación se presentan las definiciones correspondientes a redes de apoyo y cognición social, así como la relación entre áreas implicadas en cognición social que, a su vez, se han visto afectadas durante el desarrollo de la demencia, para posteriormente hacer presentación de la matriz de revisión de artículos. 


\section{Relación entre las áreas implicadas}

De acuerdo con Uribe (2010), la cognición social se define como un conjunto de procesos neurobiológicos, psicológicos y sociales mediante el cual percibimos, interpretamos, reconocemos y evaluamos el mundo social para así responder de la mejor manera posible a una situación particular. Moya, Herrero y Bernal (2010) consideran que estos procesos son cognitivo-sociales y se refieren a la forma como inferimos sobre las creencias e intenciones de otras personas, sus ventajas y desventajas de acuerdo a la situación para dar una respuesta.

Dando respuesta a uno de los objetivos específicos de la investigación, a partir de la revisión teórica se identifican las áreas cerebrales que se ven afectadas durante la demencia DTA y DFT y, a la vez, están involucradas en la cognición social. Se pueden apreciar las áreas que, por medio de la investigación de diferentes estudios, están relacionadas con las dos variables planteadas con anterioridad (ver Tabla 2).

Tabla 2.

Relación entre áreas afectadas por DTA y DFT, implicadas en cognición social

\begin{tabular}{|c|c|c|c|}
\hline Áreas & Cognición Social & DTA & DFT \\
\hline 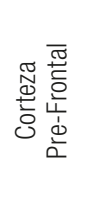 & $\begin{array}{l}\text { Esta zona se activa en el pro- } \\
\text { cesamiento de la teoría de la } \\
\text { mente. También se implica } \\
\text { en el reconocimiento de las } \\
\text { emociones faciales (Butman, } \\
\text { 2001). }\end{array}$ & $\begin{array}{l}\text { Procesos como: me- } \\
\text { moria, funciones } \\
\text { ejecutivas, toma de } \\
\text { decisiones, comporta- } \\
\text { miento, etc. (Vázquez, } \\
\text { 2008). }\end{array}$ & $\begin{array}{l}\text { Se da la comprensión de sentimientos de otras personas } \\
\text { por lo tanto una lesión causaría que falle la utilización de } \\
\text { las emociones para guiar la conducta y el sujeto actuaria no } \\
\text { en función de futuras consecuencias sino en perspectivas } \\
\text { inmediatas (Butman, 2001). }\end{array}$ \\
\hline$\frac{\frac{\pi}{0}}{\frac{\pi}{\frac{\pi}{5}}}$ & $\begin{array}{l}\text { Percepción de las emocio- } \\
\text { nes primarias y secundarias, } \\
\text { emociones faciales y teoría } \\
\text { de la mente (Butman, 2001). }\end{array}$ & $\begin{array}{l}\text { Control y recuerdo } \\
\text { de las emociones } \\
\text { (Gerring \& Zimbardo, } \\
\text { 2005). }\end{array}$ & $\begin{array}{l}\text { Procesamiento de emociones básicas y de las emociones } \\
\text { sociales que ayuda a una correcta percepción de los signos } \\
\text { sociales por lo tanto una anormalidad en esta estructura el } \\
\text { sujeto no podría inferir una emoción en otra persona y un } \\
\text { estado mental (Butman, 2001) }\end{array}$ \\
\hline 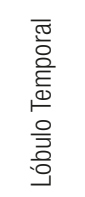 & $\begin{array}{l}\text { Se activa en la tarea de la } \\
\text { teoría de la mente (Rodríguez } \\
\text { \& Touriño, 2010). }\end{array}$ & $\begin{array}{l}\text { Memoria declarativa } \\
\text { (Solís \& López, 2009). }\end{array}$ & $\begin{array}{l}\text { Capacidad de reconocer las emociones faciales, cuyas es- } \\
\text { trategias complementarias son percepción, análisis e iden- } \\
\text { tificación y junto con ellas hay activación de las zonas de } \\
\text { corteza motora y visual que implican la representación de } \\
\text { las posturas observadas y generación del estado emocional } \\
\text { correspondiente al observado (Rodríguez \& Touriño, 2010). }\end{array}$ \\
\hline
\end{tabular}

Fuente: Elaboración Propia. DTA: Demencia Tipo Alzheimer; DFT: Demencia Fronto Temporal.

A continuación, se presenta la definición de acuerdo con referentes teóricos para redes de apoyo.

\section{Redes de Apoyo}

Para fines de esta investigación se tomará en cuenta la siguiente definición de una red social o de apoyo:
Sistema abierto a través de un intercambio dinámico entre sus integrantes y con integrantes de otros grupos, que posibilita la potenciación de los recursos que se poseen y que se enriquece con las múltiples relaciones entre los diferentes miembros que la componen (Madariaga et al., 2003, p.12). 
Las redes de Apoyo, de acuerdo con García y Madrigal (1999), están clasificadas en dos grandes grupos: a) sistemas formales: ayuda profesional por parte de una institución o de la sociedad; y b) sistemas informales: ayuda de manera no formalizada o institucionalizada, es decir, conformadas por amigos, la familia o vecinos.
De acuerdo con el objetivo principal de esta investigación, a continuación se presenta la descripción de resultados de la revisión documental, así como el modelo de la matriz utilizada para la organización de información (ver Tabla 3).

Tabla 3.

Matriz de Revisión y clasificación de las fuentes

\begin{tabular}{|c|c|c|c|c|c|c|c|c|c|c|c|c|}
\hline \multirow[b]{2}{*}{ Cita } & \multicolumn{4}{|c|}{ Características de la red } & \multicolumn{2}{|c|}{ Contextuales } & \multicolumn{3}{|c|}{ Elementos relacionales } & \multicolumn{3}{|c|}{ Condición } \\
\hline & 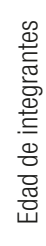 & 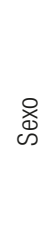 & 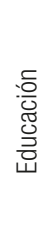 & 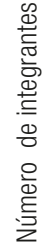 & 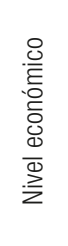 & $\begin{array}{l}\stackrel{0}{x} \\
\text { d } \\
\stackrel{0}{0} \\
0\end{array}$ & 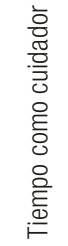 & 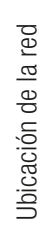 & 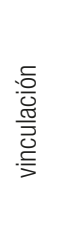 & $\begin{array}{l}\text { 胥 } \\
\text { 吾 }\end{array}$ & $\begin{array}{l}\mathscr{D} \\
\stackrel{0}{0} \\
\stackrel{0}{0} \\
\equiv\end{array}$ & 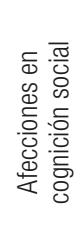 \\
\hline
\end{tabular}

Fuente: Elaboración Propia a partir de datos revisados en Cerquera et al., 2012; Cerquera y Galvis, 2014; Moreno et al., 2010.

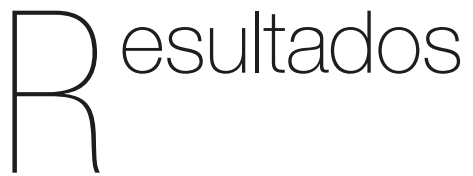

A continuación, y de acuerdo con la revisión documental realizada de 50 artículos, se presenta la descripción por factor (características de la red, contextual, elementos relacionales, condición) acompañado de la figura correspondiente (Porcentaje de Artículos que tienen en cuenta cada factor dentro de sus investigaciones).

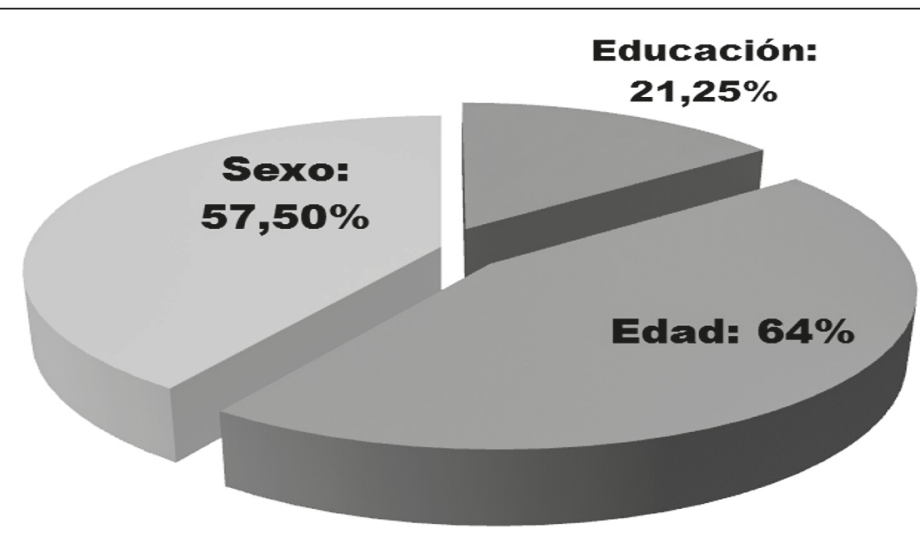

Figura 2. Resultado por características de la red

Fuente: Elaboración Propia 
En la Figura 2 se presentan los resultados obtenidos en el primer eje de la matriz documental, identificando la frecuencia con la que cada uno de estas características de la red ha sido descrita y considerada dentro las investigaciones. Dentro de la revisión documental se identificó que la edad de los cuidadores oscila entre 40-45 años, en su mayoría mujeres. El nivel educativo del cuidador varía en las diferentes investigaciones. Así mismo, en ninguna de las investigaciones revisadas se tiene en cuenta el número de integrantes en la familia (Aparicio et al., 2008; Ascher et al., 2010; Ávila, García \& Gaitán,
2015; Ayuso et al., 2007; Butman et al., 2003; Calero, Fernández \& Roa, 2009; Celdrán, Triadó \& Villar, 2009; Cerquera \& Galvis, 2013; Espín, 2012; Glynn, Hahn, Hayden, Randall \& Randolph, 2012; Kim, 2012; Krikorian, Velez, González, Palacio \& Vargas, 2010; Losada, Márquez, Puente, Gallagher, \& Knight, 2007; Mausbach, Chattillion \& Ziegler, 2012; Mausbach et al., 2014; Meyer, Nguyen, Dao, \& Arean, 2015; Mioshi, Bristow, Cook \& Hodges, 2009; Moreno, 2008; Pabón, Galvis \& Cerquera, 2014; Palacio, 2012; Valero \& Cruz, 2013; Razani, 2007; Riedjik, 2006; Rodríguez, 2013; Vargas \& Pinto, 2010)
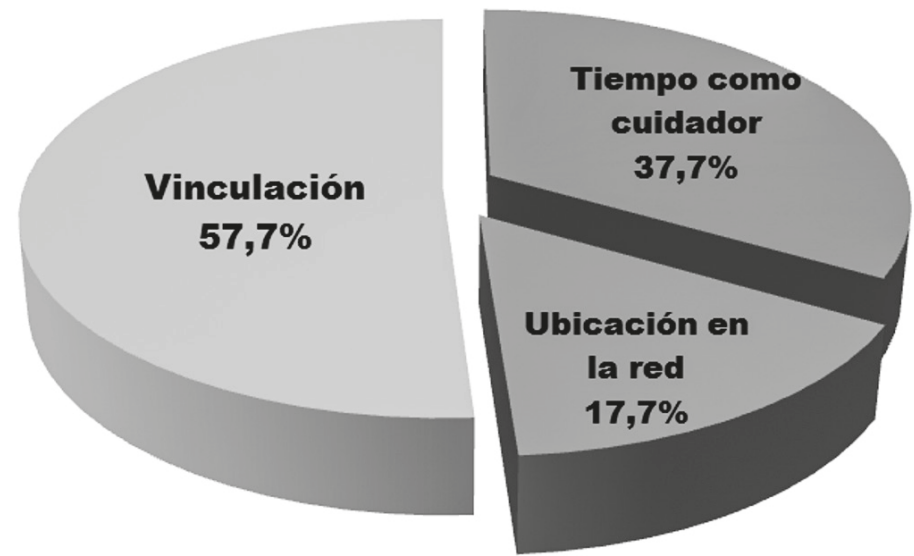

Figura 3. Resultado por elementos relacionales Fuente: Elaboración Propia.

En cuanto a elementos relacionales (ver Figura 3) se identificó que, para ser considerado cuidador principal, debe cumplir con un tiempo mínimo de 3 meses como cuidador y conviviendo con la persona. En términos de ubicación en la red, es común que el cuidador sea el cónyugue y/o hijo, en menor porcentaje se trata de personas con menor grado de consanguinidad y en su gran mayoría no se evidencia apoyo al cuidador por parte de redes informales o formales (Aparicio et al., 2008; Ávila et al., 2015; Ayuso et al., 2007; Butman et al., 2003; Calero et al., 2009; Celdrán et al., 2009; Cerquera \& Galvis, 2013; Cerquera et al., 2015; Espín, 2012; Glynn et al., 2012; Kim, 2012; Krikorian et al., 2010; Losada et al., 2007; Mausbach et al., 2012; 
Mausbach et al., 2014; Meyer et al., 2015; Moreno, 2008; Ostertag, 2014; Pabón et al., 2014; Palacio, 2012; Pérez \& Llibre, 2010; Rodríguez, 2013; Riedjik, 2006; Sánchez, Carrillo, Barrera \& Chaparro, 2013; Valero \& Cruz, 2013; Roth, Mittelman, Clay, Madan \& William, 2005; Razani, 2007; Vargas \& Pinto, 2010).

Un bajo porcentaje de investigaciones tiene en cuenta el nivel económico; así mismo, cabe resaltar que el contexto es algo que se tiene en cuenta como información mas no en términos de características que afecten los resultados (Aparicio et al., 2008; Ascher et al., 2010; Ávila et al., 2015; Ayuso et al., 2007; Butman et al., 2003; Calero et al., 2009; Celdrán et al., 2009; Cerquera \& Galvis, 2013; Cerquera et al., 2015; Espín, 2012; Kim, 2012; Glynn et al., 2012; Krikorian et al., 2010; Losada et al., 2007; Mausbach et al., 2012; Meyer et al., 2015; Moreno, 2008; Pabón et al., Galvis \& Cerquera, 2014; Palacio, 2012; Rodríguez, 2013; Roth et al., 2005; Sánchez et al., 2013; Valero \& Cruz, 2013). De acuerdo con los resultados encontrados en el tercer apartado, los datos se pueden evidenciar en la Figura 4.

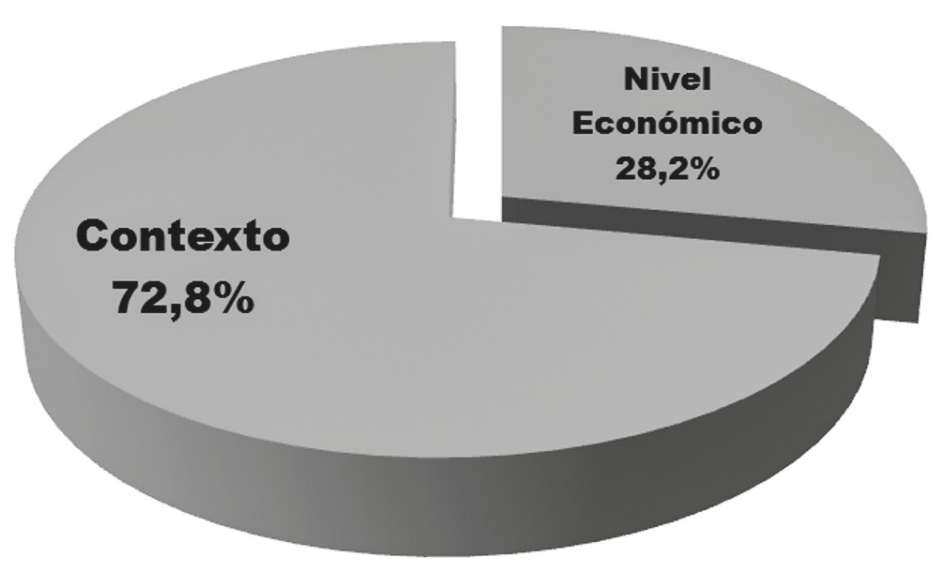

Figura 4. Resultado por características de la red

Fuente: Elaboración Propia.

Finalmente, de acuerdo con la condición del paciente (ver Figura 5), las investigaciones han considerado la clasificación del tipo de demencia. En el caso específico de la demencia tipo Alzheimer, el análisis ha incluido adicionalmente las especificidades relacionadas con la etapa de la demencia. Sin embargo, no se evidencian trabajos con análisis detenidos sobre cada una de las funciones cognitivas y neuropsicológicas afectadas, aun cuando se les menciona y describe (Butman, 2003; Celdrán et al., 2009; Espín, 2012; Glynn et al., 2012; Gramain \& Malavolti, 2004; Herrera-López et al., 2014; Losada et al., 2007; Mausbach et al., 2012; Razani, 2007; Riedjik, 2006). Tan solo el 6.6\% considera el tema de cognición social en 
el estudio de las afecciones y su impacto en el cuidador. Serrani (2013), Eslinger et al. (2007) y Fernández, Matías, Moreno y Matías (2013) afirman que el deterioro en la Demencia Frontotemporal afecta aspectos de la cognición social como teoría de la mente, evaluación de situaciones sociales, inhibición de la perspectiva propia ante el cambio, moralidad, empatía, identificación y expresión emocional. A diferencia de los pacientes con DFT, pacientes con Demencia Tipo Alzheimer tienen en un nivel menor aspectos afectados como teoría de la mente y procesamiento y reconocimiento de emociones, sin embargo, presentan dificultades para inferir la perspectiva ajena.

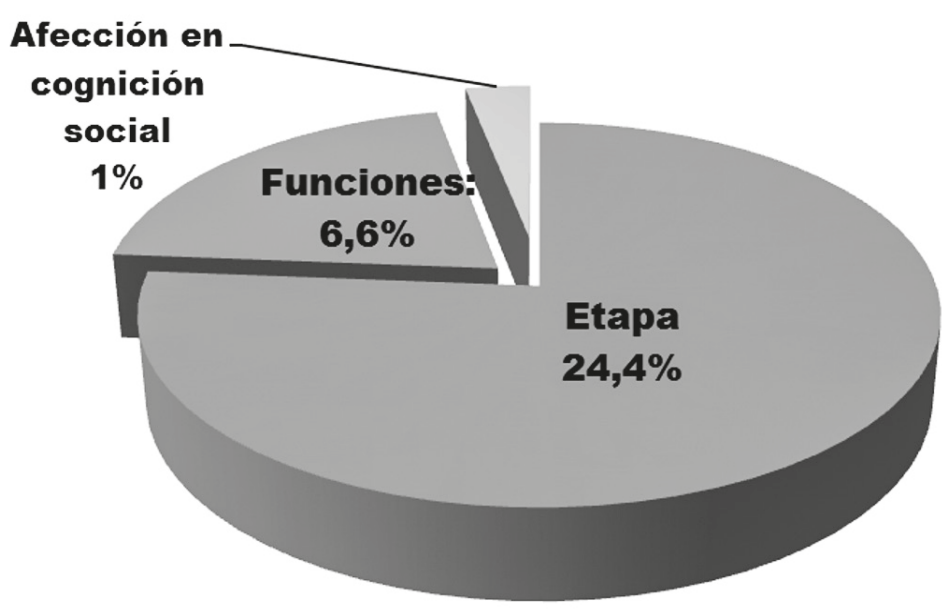

Figura 5. Resultado por características de la condición

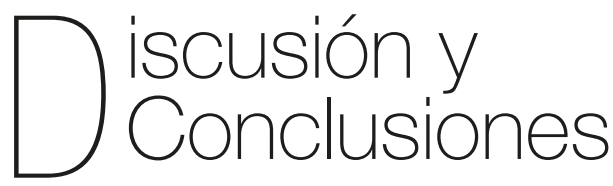

Dentro de los principales hallazgos en la comparación entre demencias se encontró que afecciones en amígdala, hipocampo y corteza prefrontal son comunes desde las explicaciones estructurales. De acuerdo con esto, y teniendo en cuenta la función de dichas estructuras, valdría la pena revisar las implicaciones a nivel emocional y de autocontrol asociadas a este tipo de demencias en quienes las padecen, dado su impacto en el entorno. Esto resulta coherente con algunos hallazgos sobre el incremento en agresividad (Krikorian et al., 2013).

A partir de este hallazgo, se encuentra que en las etapas periadolescentes afecciones en hipocampo -a propósito de posibles indicadores que permitan desarrollar investigaciones encaminadas a la búsqueda de predictores de la demencia- se desarrollan debido, entre 
otras razones, a la vivencia de situaciones de maltrato, acompañadas de estrés post-traumático (Mesa-Gresa \& MoyaAlbiol, 2011).

Dentro de los hallazgos se identifica que, entre las demencias con compromiso en cognición social, el impacto en el cuidador es de menor nivel en el caso de Demencia Tipo Alzheimer, debido quizás a que en ésta no hay compromiso emocional (Mioshi et al., 2013). Así mismo, se desconoce si participan o no las creencias y el conocimiento que tiene la red sobre las afecciones vinculadas con la demencia (Mioshi et al., 2009; Riedijk et al., 2006).

Además, se evidencia que el apoyo al cuidador por parte de otros familiares o miembros de redes informales, así como el acompañamiento de redes formales, es importante a la hora de evidenciar el impacto en el cuidador (Roth et al., 2005).

También se observa una relación inversamente proporcional entre la disponibilidad de recursos y el nivel de impacto emocional, psicológico y físico en los cuidadores (Molina, láñez \& láñez, 2005; Cerquera et al., 2012; Vargas \& Pinto, 2010; Pabón et al., 2014). Este hallazgo es coherente con lo identificado, por ejemplo, en el estudio del rol de los cuidadores y en general del papel de los integrantes del entorno social, para el caso de otras condiciones que afectan en general el funcionamiento de las personas. Este el caso, por ejemplo, desde una perspectiva orientada al estudio de la salutogenesis y, por lo tanto, de la promoción del bienestar de los cuidadores de niños con dificultad motora en quienes tambien se observan afectaciones en los indicadores emocionales y psicológicos, evaluados desde variables salutogénicas, siendo menores las puntuaciones en cuidadores de niños con estas dificultades (Vinaccia et al., 2016).

Se observa un vacío documental relacionado con investigaciones que tengan en cuenta la implicación de la cognición social en las Demencias, como factor de impacto en el cuidador. Sin embargo, un acercamiento lo realiza Serrani (2013), cuando en su estudio determina que el proceso degenerativo de la DFT afecta mayor cantidad de procesos asociados a cognición social, comparada con la DTA.

Para futuras investigaciones se recomienda tener en cuenta el contexto social en el que se desarrolla el cuidador y su historia personal, así como la forma en que las afecciones en cognición social en los pacientes pueden mediar esta relación con los cuidadores y la percepción de los mismos con respecto a la situación de cuidado.

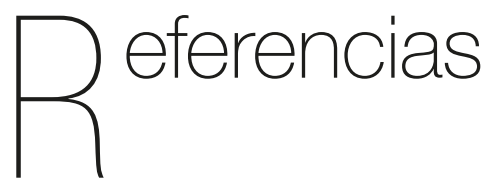

Alzheimer's Disease International (2013). Policy brief for heads of government the global impact of dementia 2013-2050. London.

Alzheimer's Disease International (2016). World Alzheimer Report 2016, Improving healthcare for people living with dementia coverage, quality and costs now and in the future. London. 
Aparicio, P., Díaz, J., Cuéllar, I., Castellanos, B., Fernández, M., \& De Tena, A. (2008). Salud Física y Psicológica de las Personas Cuidadoras: Comparación entre cuidadores formales e informales. Instituto de Mayores y Servicios Sociales. Recuperado de http://www.imserso.es/ interpresent3/groups/imserso/documents/ binario/idi24_07saludfisica.pdf

Arango, C. \& Pimienta, J. (2004). El cerebro: De la estructura y la función a la psicopatología. Primera parte: Bloques funcionales. Revista Colombiana de Psiquiatría, 33(1), 102-125.

Ascher, E., Sturm, V., Seider, B., Holley, S., Miller, B., \& Levenson, R. (2010). Relationship Satisfaction and Emotional Language in Frontotemporal Dementia and Alzheimer's Disease Patients and Spousal Caregivers. Alzheimer Disease and Associated Disorders, 24(1), 49-55. doi: 10.1097/WAD.0b013e3181bd66a3

Ávila, J., García, J., \& Gaitán, J. (2015). Habilidades para el cuidado y depresión en cuidadores de pacientes con demencia. Revista Colombiana de Psicología, 19(1), 71- 84. doi: 10.15446/rcp

Ayuso, T., Ederra, M., Manubens, J., Nuin, M., Villar, D., \& Zubicoa, J. (2007). Abordaje de la demencia. Guía de actuación en la coordinación atención primaria en Neurología. Navarra, España: Servicio Navarro de Salud. Recuperado de https:// www.navarra.es/NR/rdonlyres/90E6356A73C3-4CA1-9291-834501AA4324/0/ Guiaabordajedelademencia.pdf

Brenda, S., \& Alvarado, S. (2009). Desgaste Físico y Emocional del cuidador primario en Cáncer. Instituto Nacional de Cancerología de México, 4, 39-46.
Bruna, O., \& Cullell, N. (s.f.) Las demencias: La enfermedad de Alzheimer, intervención en demencias. Huelva, España: Fundación Uszheimer - Excma.

Butman, J. (2001). La cognición social y la corteza cerebral. Revista Neurológica Argentina, 26(3), 117-122.

Butman, J., Sarasola, D., Lon, L., Serrano, C., Arizaga, R., Tarango, F., Manes, F... Allegri, R. (2003). Impacto económico de la enfermedad de Alzheimer. Revista Neurológica Argentina, 28, 16-23.

Calero, R., Fernández, C., \& Roa, J. (2009). Cuidador informal de personas mayores dependientes y estrés percibido. Intervención Psicoeducativa, 1(1), 9-19.

Celdrán, M., Triadó, C., \& Villar, F. (2009). Nietos adolescentes con abuelos con demencia: ¿la enfermedad cambia la naturaleza de la relación?. Anales de Psicología, 25(1), 172-179.

Cerquera, A., Pabón, D., \& Uribe, D. (2012). Nivel de depresión experimentada por una muestra de cuidadores informales de pacientes con demencia tipo Alzheimer. Psicología desde el Caribe, 29(2), 360-384.

Cerquera, A., \& Galvis, M. (2014). Efectos de cuidar personas con Alzheimer: un estudio sobre cuidadores formales e informales. Pensamiento Psicológico, 12(1), 149-167. doi:10.11144/Javerianacali.PPSI12-1.ecpa

Cerquera, A., Granados, F., \&Buitrago, A. (2015). Sobrecarga en cuidadores de pacientes con demencia tipo Alzheimer. Psychologia: Avances en la Disciplina, 6(1), 35-45. http:// dx.doi.org/10.21500/19002386.1169 
Charro, C., Diéguez, E., \& González, L. (2010). La demencia más psiquiátrica: Demencia Frontotemporal, variante Frontal. Psicogeriatría, 2(4), 227-232.

De la Rubia, J., Sancho, P., \& Cabañés, C. (2014). Impacto fisiológico de la musicoterapia en la depresión, ansiedad, y bienestar del paciente con demencia tipo Alzheimer: Valoración de la utilización de cuestionarios para cuantificarlo. European Journal of Investigation in Health, Psychology and Education, 4(2), 131-140. doi: 10.1989/ejihpe.v4i2.60

Defranc, O. (2011). La neurogenética en el Ecuador. Ciencia y Avance Revista Médica, 1(1), 52-55.

Diehl, J., Schmidt, E., Nunnemann, S., Riedl, L., Kurz, A., Forstil, H., Wagenpfeil, S., \& Cramer, B., (2013). Caregiver Burden and Needs in Frontotemporal Dementia. Journal of Geriatric Psychiatry and Neurology, 26(4), 221-229. doi: 10.1177/0891988713498467

Donoso A., \& Salinas C. (2009). Demencias frontotemporales: Tres casos de la variante frontal. Revista Chilena de Neuropsiquiatría, 47(4), 286-292. Doi: http://dx.doi. org/10.4067/S0717-92272009000400005

Eslinger, P., Moore, P., Troiani, V., Antani, S., Cross, K., Kwok, S., \& Grossman, M. (2006). Oops! Resolving Social Dilemmas in Frontotemporal Dementia. Journal of Neurology, Neurosurgery, and Psychiatry, 78(5), 457-460. http://dx.doi.org/10.1136/ jnnp.2006.098228

Espín, A. (2012). Factores de riesgo de carga en cuidadores informales de adultos mayores con demencia. Revista Cubana Salud Pública, 38(3), 393-402. Doi: 10.1590/ s0864-34662012000300006
Férnandez, M., Matías, J., Moreno, T., \& Matías, J. (2013). Demencia frontotemporal variante conductual: aproximación clínica y terapéutica. Revista de Neurología, 29(8), 464-472. Doi: 10.1016/j.nrl.2013.03.001

Flores, J., \& Ostrosky-Solís, F. (2008). Neuropsicología de Lóbulos Frontales Funciones Ejecutivas y Conducta Humana. Revista de Neuropsicología, Neuropsiquiatría y Neurociencias, 8(1), 47-58.

García, H., \& Madrigal, R. (1999). Redes sociales y vejez: apoyos formales e informales en el área metropolitana de Monterrey. Papeles de Población, 5(19), 217-242.

Gerring, R., \& Zimbardo, P. (2005). Psicología y vida. Naucalpan de Juárez, México: Pearson Educación.

Gil, P. \& Martín, J. (2006). Demencia. En Sociedad Española de Geriatría y Gerontología, Tratado de gerontología para residentes (pp. 173-188). Madrid, España: International Marketing \& Communication, S.A. (IM\&C).

Glynn, S., Hahn, T., Hayden, L., Randall, F., \& Randolph, E. (2012). The use of internet Technology for Psychoeducation and Support with dementia caregivers. Psychological Services, 9(2), 215-218. Doi: 10.1037/a0027056.

Gramain, A. \& Malavolti, L. (2004). Evaluating the effect of care programs for elderly persons with dementia on caregiver's wellbeing. The European Journal of Health Economic, 5(1), 6-14. Doi: http://dx.doi. org/10.1007/s10198-003-0194-1 
Granadillo, J., \& Zarante, I. (2008). Genética de la demencia fronto temporal. Revista Colombiana de Psiquiatría, 37(1), 95-113.

Herrera, L., Alcayaga, C., Torres, M., Funk, R., Bustamante, C., Riquelme, G., Campos, S... Lange, I. (2014). Programa de apoyo al trabajador-cuidador familiar: fenómeno social emergente. Aquichan, 14(3), 430-439. http://dx.doi.org/10.5294/ aqui.2014.14.3.12

Iragorri, Á. (2007). Demencia frontotemporal. Revista Colombiana de Psiquiatría, 36(1), 139-156.

Kim, H. (2012). Predictors of caregiver burden in caregivers of individuals with dementia. Journal of Advanced Nursing, 68(4), 846-855. Doi: 10.1111/j.1365-2648.2011.05787.x.

Krikorian, A., Vélez, M., González, O., Palacio, C. \& Vargas, J. (2010). La experiencia de sufrimiento en cuidadores principales de pacientes con dolor oncológico y no oncológico. Avances en Enfermería, 28(1), 13-20.

Llibre, J. (2012). Envejecimiento y demencia: Implicaciones para la comunidad científica, la salud pública y la sociedad cubana. Anales de la Academia de Ciencias de Cuba, 2(2), 1-18.

Llibre, R., \& Gutierrez, R. (2014). Demencias y enfermedad de Alzheimer en América Latina y el Caribe. Revista Cubana Salud Pública, 40(3), 378-387

Lopera, F. (2012). Enfermedad de Alzheimer Familiar. Revista de Neuropsicología, Neuropsiquiatria y Neurociencias, 12(1), 163-188.
Losada, A. Márquez, M., Puente C., Gallagher, D., \& Knight, B. (2007). Reflexiones en torno a la atención a los cuidadores informales de personas con demencia y propuesta de una intervención interdisciplinar. Psicología Conductual, 15(1), 57-76.

Madariaga, C., Abello, R. \& Sierra, O. (2003). Redes sociales infancia, familia y Comunidad. Barranquilla, Colombia: Ediciones Uninorte

Mausbach, B., Chattillion, E., \& Ziegler, M. (2012). A Longitudinal Analysis of the Relations Among Stress, Depressive Symptoms, Leisure Satisfaction, and Endothelial Function in caregivers. Health Psychology, 31(4), 433-440. Doi: 10.1037/ a0027783

Mausbach, B., Chattillion, E., Flynn, L., Patterson, T., Tiznado, D., \& Von Kanel, R. (2014). Why does placement of persons with alzheimer's disease into long - term care improve caregivers well-being? Examination of psychological mediators. Psychology and Aging, 29(4), 776-786. Doi: 10.1037/a0037626

Méndez, L. (2010). Demencias Frontotemporales: Casos ilustrativos de las diferentes presentaciones de una misma enfermedad. Acta Neurológica Colombiana, 26(3), 1-28.

Mesa-Gresa, P. \& Moya-Albiol, L. (2011). Neurobiología del Maltrato Infantil: el ciclo de la violencia. Revista de Neurología, 52(8), 489-503.

Meyer, O., Nguyen, K., Dao, T., \& Arean, P. \&. (2015). The sociocultural context of caregiving experiences for vietnamese Dementia Family Caregivers. Asian 
American Journal of Psychology, 6(3), 263272. Doi: 10.1037/aap0000024

Ministerio de Salud (2017). Boletín de Salud Mental Demencia, subdirección de enfermedades no trasmisibles. Bogotá, Colombia.

Mioshi, E., Bristow, M., Cook, R., \& Hodges, J. (2009). Factors Underlying Caregiver Stress in Frontotemporal. Dementia and Alzheimer's Disease. Dementia and Geriatric Cognitive Disorders, 27(1), 76-81. Doi: 10.1159/000193626

Mioshi, E., Foxe, D., Leslie, F., Savage, S., Hsieh, S., Miller, L., \& Piguet, O. (2013). The impact of dementia severity on caregiver burden in frontotemporal dementia and Alzheimer disease. Alzheimer Disease \& Associated Disorders, 27(1), 68-73. Doi: 10.1097/WAD.0b013e318247a0bc

Molina, J., láñez, M., \& láñez, B. (2005). El apoyo social como modulador de la carga del cuidador de enfermos de Alzheimer. Psicología y Salud, 15(1), 33-43.

Moreno, J., Arango, J. \& Rogers, H. (2010). Necesidades familiares y su relación con las características psicosociales que presentan los cuidadores de personas con demencia. Psicología desde el Caribe, (26), 1-35.

Moreno, A. (2008). Repercusión de la enfermedad de Alzheimer en el núcleo familiar. Revista Electrónica de Psicología Social, (16), 1-14. Recuperado de http:// funlam.edu.co/revistas/index.php/poiesis/ article/viewFile/248/237

Moya, L., Herrero, N., \& Bernal M. (2010). Bases neuronales de la empatía. Revista de Neurología, 50(2), 89-100.
Ostertag, F. (2014). Soporte Social en el cuidador primario de una persona con Alzheimer. Ajayu Órgano de Difusión Científica del Departamento de Psicología UCBSP, 12(1), 79-99.

Ostrosky, F., \& Lozano, A. (2012). Demencia Frontotemporal: Una revisión. Revista Neuropsicología, Neuropsiquiatría y Neurociencias, 12(1), 189-202.

Oviedo, D., Britton, G., \& Villareal, A. (2016). Deterioro cognitivo leve y enfermedad de Alzheimer: Revisión de conceptos. Revista Investigación y Pensamiento Crítico, 4(2), 64-81.

Pabón, D., Galvis, M., \& Cerquera, A. (2014). Diferencias en el apoyo social percibido entre cuidadores informales y formales de pacientes con demencia tipo Alzheimer. Psicología desde el Caribe, 31(1), 59-78. http://dx.doi.org/10.14482/psdc.31.1.5339

Palacio, K. (2012). Cognición y Conducta Social en una muestra de Pacientes Colombianos con la Variante Frontal de la Demencia Frontotemporal (DFTvf). (Tesis de Maestría). Universidad Nacional, Bogotá, Colombia.

Pérez, M. (2005). Demencia en la enfermedad de Alzheimer: un enfoque integral. Revista Cubana de Medicina General Integral, 21(3-4). Recuperado de http://scielo.sld. $\mathrm{cu} /$ scielo.php?script=sci_arttext\&pid $=$ S0864-21252005000300017

Pérez, M. (2008). Las intervenciones dirigidas a los cuidadores de adultos mayores con enfermedad de Alzheimer. Revista Habanera de Ciencias Médicas, 7(3). Recuperado de http://scielo.sld.cu/scielo. php? script=sci_arttext\&pid=S1729519X2008000300009 
Pérez, M., \& Llibre, J. (2010). Características sociodemográficas y nivel de sobrecarga de cuidadores de ancianos con Enfermedad de Alzheimer. Revista Cubana de Enfermería, 26(3), 110-122.

Razani, J. (2007). Predicting Caregiver Burden from Daily Functional Abilities of. Journal of the American Geriatrics Society, 55(9), 1415-1420. Doi: 10.1111/j.1532-5415.2007.01307.x

Rivas, M. (2008). Procesos cognitivos y aprendizaje significativo. Madrid, España: Subdirección General de Inspección Educativa de la Viceconsejería de Organización Educativa de la Comunidad de Madrid.

Riedijk, S., De Vugt, M., Duivenvoorden, H., Van Swieten, J., Verhey, F., \& Tibben, A. (2006). Caregiver Burden, Health Related Quality of Life and Coping in Dementia Caregivers: A comparison of Frontotemporal Dementia and Alzheimer's Disease. Dementia and Geriatric Cognitive Disorders, 22(5-6), 405-412. Doi: 10.1159/000095750

Rodríguez, C. (2013). Las familias y los cuidados a las personas mayores dependientes: entre la reciprocidad y la ambivalencia. Cuadernos de Trabajo Social, 26(2), 349-358. Doi: http:// dx.doi.org/10.5209/rev_CUTS.2013.v26. n2.42291

Rodríguez, J., \& Touriño, R. (2010). Cognición social en la esquizofrenia: Una revisión del concepto. Archivos de Psiquiatría, 73(9), 1-19.

Roth, D., Mittelman, M., Clay, O., Madan, A., \& William, E. (2005). Changes in
Social Support as Mediators of the Impact of a psychosocial Intervention for Spouse Caregivers of Persons with Alzheimer's Disease. Psychology and Aging, 20(4), 634-644. Doi: 10.1037/0882-7974.20.4.634

Ruiz, M. (2003). Reseña de "Manual de Neurogenética" de Adriano Jiménez Escrig. Revista de la Asociación Española de Neuropsiquiatría, (86), 125-126.

Ruiz, C., Nariño, D., \& Muñoz, J. (2010). Epidemiología y carga de la enfermedad de Alzheimer. Acta Neurológica Colombiana, 26(3), 87-94

Sánchez, B., Carrillo, G. M., Barrera, L., \& Chaparro, L. (2013). Carga del cuidado de la enfermedad crónica no transmisible. Aquichan, 13(2), 247-260.

Setó-Salvia, N. \& Clarimón J. (2010). Genética en la enfermedad de Alzheimer. Revista de Neurología, 50, 360-400.

Solís H., \& López, E. (2009). Neuroanatomía funcional de la memoria. Archivos de Neurociencia, 14(3), 176-187

Uribe, C. (2010). Una breve introducción a la cognición social: Procesos y estructuras relacionados. Revista Virtual de Psicología, Contextos, (4), 1-10. Recuperado de http://www.contextosrevista.com.co/Revista\%204/A5_ Una\%20introduccion\%20a\%20la\%20 cognicion\%20social.pdf

Valero, A. \& Cruz, N. (2013). La familia ante la enfermedad de Alzheimer. La problemática de los grupos vulnerables, visiones de la realidad. Tomo IV. (pp. 1127). Coahuila: México. 
Vargas, L \& Pinto, N. (2010). Calidad de vida del cuidador familiar y dependencia del paciente con Alzheimer. Avances en Enfermería, 28(1), 116-128.

Vázquez, P. (2008). Proyecciones de la corteza prefrontal a los núcleos monoaminergicos del mesencéfalo: vías y receptores implicados. (Tesis Doctoral). Universidad de Barcelona: Barcelona. Recuperado de http://diposit.ub.edu/dspace/ bitstream/2445/36039/1/PEVB_TESIS.pdf

Vinaccia, S., Quiceno, J., Salcedo, M., González, M., Henao, J., \& Herrera, C. (2016). Factores salutogenicos y patogenicos en padres de niños saludables con dificultad motora. Informes Psicológicos, 16(1), 101-115. 